Document downloaded from:

http://hdl.handle.net/10251/78852

This paper must be cited as:

Calabuig, JM.; Ferrer Sapena, A.; Sánchez Pérez, EA. (2016). Vector-valued impact measures and generation of specific indexes for research assessment. Scientometrics. 108(3):1425-1443. doi:10.1007/s11192-016-2039-6.

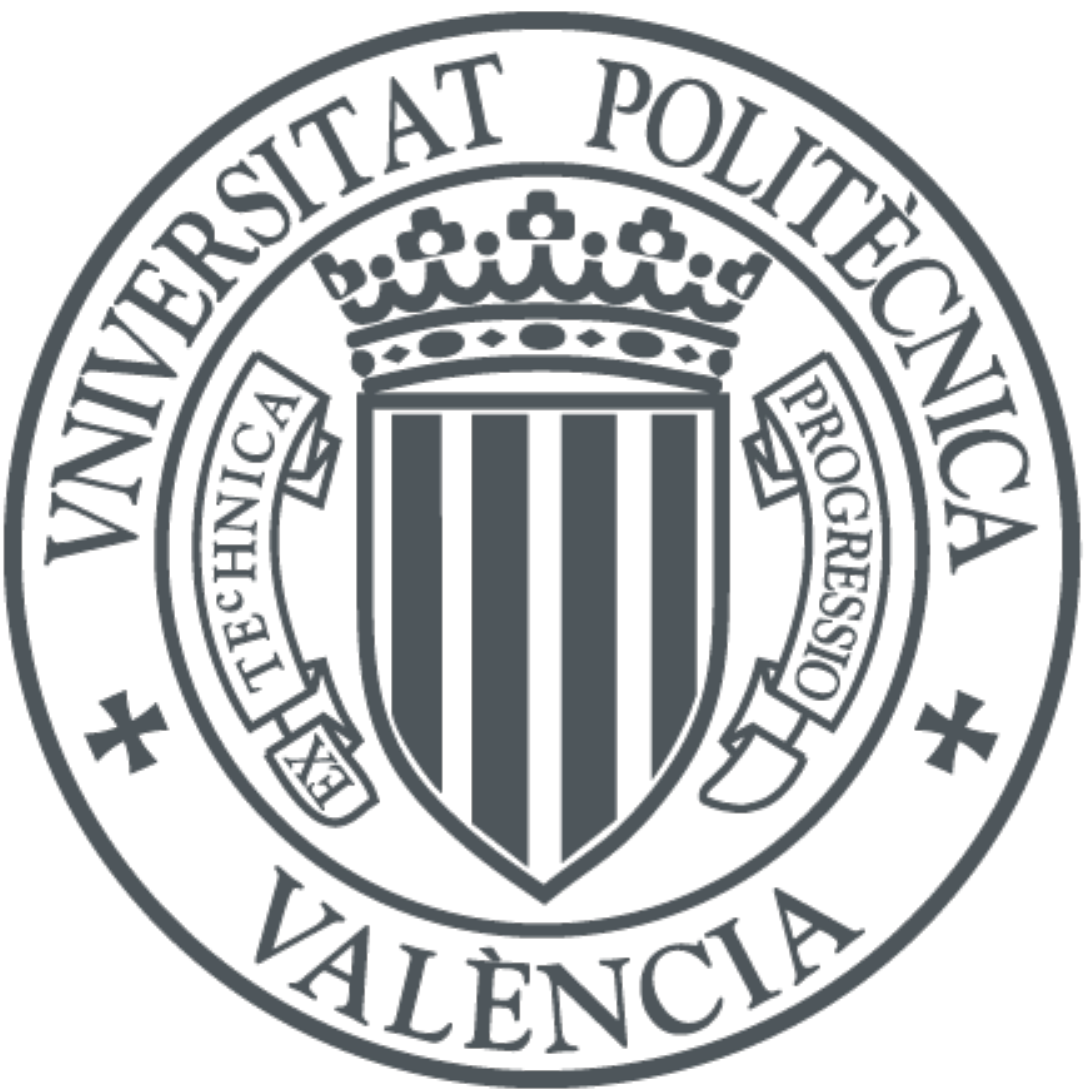

The final publication is available at

http://dx.doi. org/10.1007/s11192-016-2039-6

Copyright Akadémiai Kiadó; Springer

Additional Information 


\title{
VECTOR-VALUED IMPACT MEASURES AND GENERATION OF SPECIFIC INDEXES FOR RESEARCH ASSESSMENT
}

\author{
J.M. CALABUIG, A. FERRER-SAPENA, AND E.A. SÁNCHEZ-PÉREZ
}

\begin{abstract}
A mathematical structure for defining multi-valued bibliometric indices is provided with the aim of measuring the impact of general sources of information others than articles and journals - for example, repositories of datasets-. The aim of the model is to use several scalar indices at the same time for giving a measure of the impact of a given source of information, that is, we construct vector valued indices. We use the properties of these vector valued indices in order to give a global answer to the problem of finding the optimal scalar index for measuring a particular aspect of the impact of an information source, depending on the criterion we want to fix for the evaluation of this impact. The main restrictions of our model are 1) it is based in the use of a finite set of scalar impact indices (altmetrics), and 2) these indices are assumed to be additive. The optimization procedure for finding the best tool for a fixed criterion is also presented. In particular, we show how to create an impact measure completely adapted to the policy of an specific research institution.

Impact factor and vector valued and model and mathematics and altmetrics. PAC: C20, C65 and C80, AMS Class: 94A15 and 94A17 and 28E99
\end{abstract}

\section{INTRODUCTION}

Nowadays, impact measuring tools for information sources are everywhere as principal elements for a lot of relevant decisions almost in every aspect of social life. For instance, an everyday action done by millions of people in the world -as making a Google search- is based on an algorithm for ordering the list of results by the "prestige" or "impact" of each of them. Regarding scientific information "objects" — as papers, journals or databases - to find good mathematical instruments for measuring the impact and estimate the "quality" of a given object from it is an increasingly demanding challenge (Aleixandre Benavent et al. 2007). The main application of these tools is probably research assessment of public scientific institutions, universities and technological companies, as well as being a relevant for research groups for obtaining the specialized information that they need (BuelaCasal 2003; see also Ferrer-Sapena et al. (preprint) and the references therein).

Indeed, estimating the relevance of scientific documents has become a fundamental tool for managing the scientific activity. It concerns the design of the policy of public and private institutions, and has decisive consequences in the scientific research, and also in other relevant social activities. A concrete fact that shows how important measuring the impact of the scientific information is, is the great development of the technical tools used for measuring the impact of the scientific papers. The (2-year) Impact Factor of Thomson-Reuters or the SJR Scimago rank are main references in this setting, but there are a lot of classical and new indices that have been applied, depending on the choice of the group of experts and the 
use that is going to be given to the tool (Egghe and Rousseau 2002; see also for example Leydesdorff and Opthof 2010). The so called altmetrics has burst onto the scene in recent years, changing the point of view that was assumed in the past that only one index is enough for measuring the impact. Nowadays, the experts agree with the idea that several indexes must be used to have a complete overview of what is going on in a certain research field.

The aim of this paper is twofold. On one hand, we will provide an abstract mathematical structure for defining what a source of information is -including papers, but also databases, public outputs of simulation programs for the forecast prediction...-, as well as an abstract definition of what a measure of impact of a subset of this source of information has in other information sets. On the other hand, we will introduce the elements that are necessary for defining a vector valued impact index. This is the natural way of providing a mathematical uniform structure for considering several indexes as parts of a whole for measuring the influence of a concrete subset. Finally, we will show how to obtain from this vector valued tool a concrete scalar valued impact factor that reflects the preferences of a given research institution according to its scientific policy.

We will use standard normed space notation in the paper. For a natural number $n$, we will write the vectors of the linear space $\mathbb{R}^{n}$ by means of its coordinates as $v=\left(x_{1}, \ldots, x_{n}\right)$. This space in assumed to be endowed with the standard Euclidean norm. Alternatively, we will call $e_{1}, \ldots, e_{n}$ to the unit vectors of the canonical basis of the space, that is, $e_{1}=(1,0, \ldots, 0), e_{2}=(0,1,0, \ldots, 0)$, and so on. Thus a vector $x \in \mathbb{R}^{n}$ will be written as

$$
v=\left(x_{1}, \ldots, x_{n}\right)=\sum_{i=1}^{n} x_{i} e_{i} .
$$

\section{VECTOR VALUED IMPACT FACTORS: THE GENERAL FRAMEWORK}

After the so called altmetrics began to be a general way for measuring information sources, there are a lot of different available indexes: of course, the choice depends on the intended use (Piwovar 2013; Zahedi et al. 2014, see also the references therein). However, it seems to be more adequate for performing a complete analysis of a given ranking problem for a fixed "information set" - group of journals, databases, repositories,...- to consider it as a multivalued problem. That is, roughly speaking the correct way is to put each index in a different coordinate, providing in this way a vector valued measure, and the final vector valued index of the "information set" is given by the vector composed by all the indexes associated to the set. This allows to use different scalar measures for an information set. The first one is given by the computation of the norm of the set, that provides a "uniform" measure of the value of all the indexes considered. The second one can be defined by the action of an element of the dual space on the vector, what

gives a scalar measure with weights provided by the coordinates of the dual. This is what we will call a determination of the associated vector valued index, and will be explained later on the paper.

The main restriction of the model that we will explain in what follows is given by the fact that all the - scalar and vector valued - set functions are countably additive. Taking into account that most of the new altmetrics that are being introduced as measures of the impact of sources of information are defined using increasing capacities — also called fuzzy measures - that are not additive, this is a 
rather strong requirement. Recall that a nonempty set $\Sigma$ consisting on subsets of a set $\Omega$ is said to be a $\sigma$-algebra if $\emptyset \in \Sigma, A^{c}=\Omega \backslash A \in \Sigma$ whenever $A \in \Sigma$ and it is closed under countable unions. A scalar set function $\mu: \Sigma \rightarrow \mathbb{R}$ is called additive when $\mu(A \cup B)=\mu(A)+\mu(B)$ whenever $A$ and $B$ are disjoint elements of $\Sigma$. If the previous equality holds for any (countable) sequence of pairwise disjoint elements in $\Sigma$-in other words, $\mu$ is countable additive - then $\mu$ is called a scalar measure. For example, some new impact indexes for journals and authors as the $h$-index and other measures that can be modeled using Choquet, Sugeno and universal integrals, are not in general additive: the reader can find information about this topic in Gagolewski and Mesiar (2014); Klement et al. (2010); Torra and Narukawa (2008); Beliakov and James (2011). However, in this paper we deal only with the additive case mainly by two reasons:

1) All the classical impact factors IF (Thomson-Reuters, weighted versions of the two year IF,...) are defined by means of additive measures (see FerrerSapena et al. (2015); Alguliyev et al. 2015; Habibzadeh and Yadollahie 2008; Waltman and van Eck 2008; Zitt and Small 2008; Zyczkowski 2010).

2) The non-additive case can be adapted from the additive one using the same construction. Of course, some of the structure results do not work, but the uniform treatment as set functions defined in vector spaces can be assumed to be the same. For example, we cannot expect the result to be a vector measure but a vector lattice valued increasing capacity (also called fuzzy vector measures); see Klement et al. (2010); Beliakov and James (2011); Pinski and Narin (1976) for how to use the scalar version of this notion.

Under these restrictions, we propose the following model. Through this section consider a fixed positive measure space $(\Omega, \Sigma, \mu)$, that is, $\Omega$ is a nonempty set, $\Sigma$ is a $\sigma$-algebra of subsets of $\Omega$ and $\mu$ is a positive measure (i.e. $\mu: \Sigma \rightarrow \mathbb{R}^{+}$is a countable additive set function). We say that a Banach space valued function $\Phi: \Sigma \rightarrow E$ is absolutely continuous with respect to $\mu$ ( $\mu$-continuous for short) if and only if $\mu(A)=0$ implies that $\Phi(A)=0, A \in \Sigma$.

The standard definition that generalizes the classical idea of impact factor is given in the following:

Consider a (countably additive) positive measure $\eta: \Sigma \rightarrow \mathbb{R}^{+}$which is absolutely continuous with respect to $\mu$. We say that the set function $I: \Sigma \rightarrow \mathbb{R}^{+}$given by

$$
I(A):=\frac{\eta(A)}{\mu(A)}, \quad A \in \Sigma
$$

is a scalar impact measure with respect to $\mu$.

This notion can be extended to the vector valued setting as follows. Let $E$ be a (real) Banach space. As in the scalar case, a vector valued set function $m: \Sigma \rightarrow E$ is said to be a vector measure when $m$ is countable additive - that is $m\left(\bigcup_{k=1}^{\infty} A_{k}\right)=$ $\sum_{k=1}^{\infty} m\left(A_{k}\right)$ for each sequence of pairwise disjoint $A_{1}, A_{2}, \ldots \in \Sigma-$.

Let $E$ be a (real) Banach space. A $\mu$-continuous set valued function $I: \Sigma \rightarrow E$ is said to be a vector valued impact measure with respect to $\mu$ if $\Phi(\cdot):=I(\cdot) \mu(\cdot)$ is a vector measure.

Additivity of the resulting vector valued function is not fundamental for the optimization process we propose in the following section. However, it is convenient for the purpose of representation, since - as we will show - each $\mu$-continuous measure can be represented as a Lebesgue integral with some scalar weight. Therefore, we 
will see that for the finite dimensional case, all the additive vector valued impact measures can in fact be written as compositions of weighted scalar measures. In the following definition we use the notion of Bochner integrable function, that is the direct extension of Lebesgue integrable function for vector valued measures. We write $L^{1}(\mu, E)$ for the space of all $E$-valued Bochner integrable functions. The reader can find in the book by Diestel and Uhl (1977) the definition and basic properties.

We say that an impact measure $I: \Sigma \rightarrow E$ defined with respect to $\mu$ is $a$ representable impact measure if there is a Bochner integrable function $f_{0} \in L^{1}(\mu, E)$ such that

$$
I(A):=\frac{1}{\mu(A)} \int_{A} f_{0} d \mu, \quad A \in \Sigma .
$$

Consider $\Omega$ to be the set of all the articles that were published in 2010 in all the journals appearing in the JCR subject MATHEMATICS. Let $\Sigma$ the set algebra defined by all the subsets of $\Omega$. Define the functions $N_{i}: \Omega \rightarrow \mathbb{R}, i=1,2,3,4$,

$$
\begin{aligned}
N_{i}(a):= & \text { number of citations to the paper " } a \text { " from papers published } \\
& \text { in the year } 201 i \text { in journals in the same JCR list, }
\end{aligned}
$$

and write $\mu: \Sigma \rightarrow \mathbb{R}^{+}$to be the counting measure for any set $A$, that is, $\mu(A)=|A|$, the cardinal of $A$. The indexes we want to consider in the vector valued impact measure are

$$
\phi_{i}(A)=\sum_{a \in A} N_{i}(a)
$$

$i=1,2,3,4$, that is the number of citations to a set of articles $A$ published in 2010 in the year 201i. Let us consider the set function $I: \Sigma \rightarrow \mathbb{R}^{4}$, defined by

$$
I(A):=\frac{1}{\mu(A)}\left(\phi_{1}(A), \phi_{2}(A), \phi_{3}(A), \phi_{4}(A)\right), \quad A \in \Sigma,
$$

that can be written - taking into account that in this case integration is just summation- as

$$
\begin{aligned}
I(A) & =\frac{1}{\mu(A)} \int_{A}\left(\phi_{1}(a), \phi_{2}(a), \phi_{3}(a), \phi_{4}(a)\right) d \mu(a) \\
& =\frac{1}{\mu(A)} \int_{A}\left(\sum_{i=1}^{4} \phi_{i}(a) e_{i}\right) d \mu(a) .
\end{aligned}
$$

Thus, in this case $f_{0}=\sum_{i=1}^{4} \phi_{i} e_{i} \in L^{1}\left(\mu, \mathbb{R}^{4}\right)$. Of course, the relevant values of these functions are the ones that it takes when the set $A$ is defined to be the set of all articles published in a given journal in the year 2010. However, notice that the definition makes sense for any subset of articles in $\Omega$.

The vector valued weight appearing in (1) may play also the role of relative normalization among the different components (coordinates) of the final impact measure. In this sense, it provides a suitable way of modeling two important facts that involve the use of classical impact factors as an assessment tool: normalization and comparison among different scientific fields, see Moed (2010); Zitt (2011); Dorta-González and Dorta-González (2013); Dorta-González et al. (2014); Li et al. (2013); Owlia et al. (2011); Ruiz Castillo and Waltman (2015). Thus, the vector valued function in (1) may contain the weights that we want to give to 
each coordinate appearing in the vector valued measure. Let us show that in the following

Let us show a 2-dimensional valued impact measure. Consider the set $\mathcal{R}$ of all the journals in a given subject of the Journal Citation Reports of Thomson-Reuters of the year 2014. We want to define a mixed vector valued index with the 2-year Impact Factor $\mathrm{IF}_{2}$ and the 5-year Impact Factor $\mathrm{IF}_{5}$ acting on this set of journals, that are the elements of the set for which the index is defined. However, we want the influence in the index of $\mathrm{IF}_{2}$ to be double than the influence of $\mathrm{IF}_{5}$. We consider the $\sigma$-algebra $\Sigma$ of all the subsets of journals in $\mathcal{R}$. We want to define a vector valued impact measure $I: \Sigma \rightarrow \mathbb{R}^{2}$ that satisfies that for every single journal $r \in \mathcal{R}$,

$$
I(\{r\}):=\left(2 \cdot \mathrm{IF}_{2}(r), \mathrm{IF}_{5}(r)\right) .
$$

Let us show that this can be written as a vector valued impact measure according to Definition 2. Note first that the elements of $\Sigma$ are finite sets of journals. Define the scalar atomic measure $\mu$ acting in $\Sigma$ by

$$
\mu(R)=\sum_{r \in R} n_{2}(r), \quad R=\left\{r_{1}, r_{2}, \ldots, r_{n}\right\} \in \Sigma,
$$

where $n_{2}(r)$ is the number of papers published by the journal $r$ in 2012 and 2013 . Therefore, we can write $I$ as

$$
\begin{aligned}
I(R) & =\left(\frac{1}{\mu(R)} \sum_{r \in R} 2 \cdot \mathrm{IF}_{2}(r) \cdot \mu(r), \frac{1}{\mu(R)} \sum_{r \in R} \operatorname{IF}_{5}(r) \cdot \mu(r)\right) \\
& =\frac{1}{\mu(R)}\left(\int_{R} 2 \cdot \mathrm{IF}_{2}(r) d \mu(r), \int_{R} \mathrm{IF}_{5}(r) d \mu(r)\right) \\
& =\frac{1}{\mu(R)} \int_{R}\left(2 \cdot \mathrm{IF}_{2}(r) e_{1}+\mathrm{IF}_{5}(r) e_{2}\right) d \mu(r),
\end{aligned}
$$

for $R \in \Sigma$. So, in this case $f_{0}=2 \cdot \mathrm{IF}_{2} e_{1}+\mathrm{IF}_{5} e_{2} \in L^{1}\left(\mu, \mathbb{R}^{2}\right)$.

Actually Definition 2 involves one of the most relevant notions in modern functional analysis: a Banach space $E$ has the Radon-Nikodým property if for each $\mu$-continuous vector measure $m: \Sigma \rightarrow E$ of bounded variation there is a Bochner integrable function $f_{0} \in L^{1}(\mu, E)$ such that $m(A)=\int_{A} f_{0} d \mu, A \in \Sigma$; see for example Section III.1 in Diestel and Uhl (1977). Therefore, under the hypothesis that $E$ has the Radon-Nikodým property, each countably additive vector valued impact measure with respect to $\mu$ is representable, in the sense that there is a function $f_{0} \in L^{1}(\mu, E)$ that can be identified with the vector valued Radon-Nikodým derivative - denoted by $d(I \mu) / d \mu-$, such that

$$
I(A)=\frac{1}{\mu(A)} \int_{A}\left(\frac{d(I \mu)}{d \mu}\right) d \mu=\frac{1}{\mu(A)} \int_{A} f_{0} d \mu, \quad A \in \Sigma .
$$

Recall that for scalar measures $\nu$, this property always holds as a consequence of the classical Radon-Nikodým theorem, that is one of the cornerstones of the measure theory: if $\nu$ is $\mu$-continuous, then there is a Lebesgue integrable function $f \in L^{1}(\mu)$ such that $\nu(A)=\int_{A} f d \mu, A \in \Sigma$. In other words, every finite dimensional Banach space $E$ has the Radon-Nikodým property, since if $\operatorname{dim}(E)=n$, we can apply this result for each coordinate projection $\nu_{i}(\cdot)=\left\langle I \mu(\cdot), e_{i}\right\rangle$ for obtaining a scalar Radon-Nikodým derivative $f_{i}$, and then put all of them together as $f=\sum_{i=1}^{n} f_{i} e_{i}$ 
for giving the required Bochner integrable function. This will be used in the next section.

\section{The Standard CASE: Finite Dimensional IMPaCt MEASURES}

Let $I_{1}, \ldots, I_{n}$ be a finite set of scalar impact measures that are defined by means of positive measures $\eta_{1}, \ldots, \eta_{n}$, all of them being absolutely continuous with respect to a fixed scalar measure $\mu$. Consider the vector valued set function $I: \Sigma \rightarrow\left(\mathbb{R}^{n}\right)^{+}$ given by

$$
I(A):=\frac{1}{\mu(A)} \sum_{i=1}^{n} \eta_{i}(A) e_{i}, \quad A \in \Sigma .
$$

Let $I$ defined as above.

(i) $I$ is defined by a positive vector measure. More concretely the set function $m: \Sigma \rightarrow\left(\mathbb{R}^{n}\right)^{+}$given by $m(A):=\mu(A) I(A)$ defines such a vector measure.

(ii) There are functions $f_{i} \in L^{1}(\mu)$ such that

$$
I(A)=\frac{1}{\mu(A)} \int_{A}\left(\sum_{i=1}^{n} f_{i} e_{i}\right) d \mu, \quad A \in \Sigma .
$$

In other words, there is a non-negative Bochner integrable function $f \in$ $L^{1}\left(\mu, \mathbb{R}^{n}\right)$ such that $I(A)=\int_{A} f d \mu / \mu(A), A \in \Sigma$.

Proof. (i) is obvious. For (ii), just use the Radon-Nikodým Theorem for the positive scalar measures $\eta_{i}$ which coincide with $\left\langle m, e_{i}\right\rangle(A):=\left\langle m(A), e_{i}\right\rangle, A \in \Sigma, i=1, \ldots, n$, for obtaining the required Bochner integrable function, as it is explained in Remark 2 .

The functions $f_{i}, i=1, \ldots, n$ play the role of the weights that appear in a natural way when a scalar impact factor is adapted to particular situations. The main example is given by the case when the 2-year impact factor is wanted to be weighted in order to change the value of each citation in the final sum. For example, when we want to distinguish among citations made by papers in very prestigious journals and citations coming from normal journals, making the first group of cites to weight double than the second one. Many other examples of weighted impact factors can be given - see Ferrer et al. (2015) and the references therein-.

Once the vector valued vector measure is defined and a functional representation $A \rightsquigarrow \int_{A} f d \mu / \mu(A) \in\left(\mathbb{R}^{n}\right)^{+}$is obtained, we are interested in representing the corresponding scalar components of the vector. Although our results can be easily adapted to the general framework of the Banach lattices - in that case the components are the values of the vector valued measure when an element of the dual space acts over it - since in this work our Banach lattice is $E=\mathbb{R}^{n}$ we prefer to restrict ourselves to this concrete setting. As usual, by $(\cdot \mid \cdot)$ we denote the classical Euclidean scalar product in $\mathbb{R}$.

Let $I_{f, \mu}$ be a vector valued impact measure associated to a non-negative function $f: \Omega \rightarrow\left(\mathbb{R}^{n}\right)^{+}$and a probability measure $\mu: \Sigma \rightarrow \mathbb{R}$-i.e. $\mu$ is a scalar measure satisfying $\mu(\Omega)=1$ - For each norm one $x^{*} \in\left(\mathbb{R}^{n}\right)^{+}$we will say that $\left(I_{f, \mu}\right)_{x^{*}}$ defined by

$$
\left(I_{f, \mu}\right)_{x^{*}}(A):=\left(I_{f, \mu}(A) \mid x^{*}\right)=\frac{1}{\mu(A)}\left(\int_{A} f d \mu \mid x^{*}\right), \quad A \in \Sigma
$$


is a determination of $I_{f, \mu}$. It can be easily noted that each determination defines a (positive) scalar impact measure.

In our model, we want the action of such an element $x^{*}$ on $I_{f, \mu}$-i.e., the determination of $I_{f, \mu}$ given by $x^{*}$-, to represent the weight the evaluator wants to give to each one of the impact factors composing the vector valued impact measure $I_{f, \mu}$. Let us provide a concrete example.

Suppose that the vector valued impact measure $I$ is composed for each $A \in \Sigma$ by the 5 -fold vector

$$
I(A):=\left(I_{0}(A), I_{1}(A), I_{2}(A), I_{3}(A), I_{4}(A)\right) \in\left(\mathbb{R}^{n}\right)^{+} .
$$

For the aim of simplicity, let us assume in this case that $\mu$ is the counting measure $c$ on the finite set of all the articles published in journals of the list of MATHEMATICS in 2014, and $f$ is the constant function equal to 1 . That is, $I:=I_{1, c}$. Here, $I_{0}, I_{1}, I_{2}, I_{3}$ and $I_{4}$ are defined to be the Thomson-Reuters 2-year impact factors of the subject MATHEMATICS for the years 2010, 2011, 2012, 2013 and 2014, respectively. Suppose that we want to define a new impact factor say CI for the year 2014 as a cumulative index, taking into account the values of the 2-years IF's of the last 5 years in order to provide a smoother behavior for the final index. Take the decreasing set of weights given by the norm one element $x_{0}^{*}=(0.1,0.3,0.4,0.5,0.7) \in\left(\mathbb{R}^{5}\right)^{+}$. Then the determination of $I$ that fits with our aim is given by

$$
I_{x_{0}^{*}}(A)=\left(I(A) \mid x_{0}^{*}\right)=0.1 I_{0}(A)+0.3 I_{1}(A)+0.4 I_{2}(A)+0.5 I_{3}(A)+0.7 I_{4}(A),
$$

for $A \in \Sigma$. For example, this cumulative index for the journal Acta Mathematica Hungarica is computed as follows. Consider the set $H$ of $\Sigma$ given by all the papers published in the journal in 2015. The values of the impact factor for Acta Mathematica Hungarica for the years 2010 to 2014 are $0.521,0.456,0.348,0.401$ and 0.429 , respectively. Then the value of the cumulative index for this journal in 2014 is

$$
I_{x_{0}^{*}}(H)=0.1 \cdot 0.521+0.3 \cdot 0.456+0.4 \cdot 0.348+0.5 \cdot 0.401+0.7 \cdot 0.429=0.8289 \text {. }
$$

The reader can find more information, applications and examples of this kind of cumulative indexes in Ferrer et al. (preprint).

\section{Performing the Best Determination for a finite dimensional impact MEASURE}

As we explained in the introduction, the problem we want to face in this paper is to give an optimal scalar impact measure for a concrete evaluation process using a finite set of scalar impact measures that we want to be involved. For example, we want to find the best scalar impact measure using the 2-year impact factors of the last 5 years, as in Example 3. However, we do not want to fix the coefficients that define $x_{0}^{*}$ in this example. We are interested in finding the ones that provide the best scalar impact measure for ordering the importance of the sets of information included in $\Sigma$, according to a fixed criterium. The final aim is to get a suitable tool for ordering the information sets of $\Sigma$ - for example, journals - by their "relevance" by means of an optimization procedure.

The optimality of the coefficients will be given by the following rule: we choose a concrete finite set of information sets in $\Sigma$ that we want to be well-evaluated. In other words, we want them to have as good marks as possible, since we know that they represent "high quality" information sources. In the example of journals, 
we take a set of journals that we want to be "on the top" of our list, whenever the scalar indexes used allow that. For example, if we are using the IF of a list of MATHEMATICS, choosing the subset of the journals in Algebra for having as good marks as possible, should provide a specific index for the subarea of MATHEMATICS defined as Algebra.

With this aim, we develop in what follows the mathematical procedure for performing such method for providing the solution to the following

Problem: Take a finite set of scalar impact measures $I_{1}, \ldots, I_{n}$ and take a finite list of elements $A_{1}, \ldots, A_{m}$ of $\Sigma$. Find the determination that provides the highest values for the selected sets $A_{1}, \ldots, A_{m}$ among all the determinations of the vector valued impact measure given by $I=\left(I_{1}, I_{2}, \ldots, I_{n}\right)$.

The optimization problem is solved as follows. The function we want to attain its maximum value is

$$
\Psi\left(x^{*}\right)=\left(I\left(A_{1}\right) \mid x^{*}\right)+\ldots+\left(I\left(A_{m}\right) \mid x^{*}\right),
$$

that is, the sum of all the contributions of the selected sets to the evaluation of the determination of the vector valued impact measure. The variables are the coefficients of the norm one vectors $x^{*}:=\left(\lambda_{1}, \ldots, \lambda_{n}\right) \in\left(\mathbb{R}^{n}\right)^{+}$. The solution is the (norm one positive) vector $x_{0}^{*}$ in which the function $\Psi$ attains the maximum. Recall that we are considering the 2 -norm for $\mathbb{R}^{n}$.

The function is continuous with respect to the norm; in fact, it is defined by a functional in the dual space $\left(\mathbb{R}^{n}\right)^{*}$. Therefore, elementary calculus proves that the maximum is attained for an element of the norm compact set

$$
\mathcal{C}=\left\{\left(\lambda_{1}, \ldots, \lambda_{n}\right) \in\left(\mathbb{R}^{n}\right)^{+}: \sum_{i=1}^{n} \lambda_{i}^{2}=1\right\}
$$

Moreover, since $\left(\mathbb{R}^{n},\|\cdot\|_{2}\right)$ is a Hilbert space, it is smooth, and so the solution is unique — see for example Part 3 in Beauzamy (1982)-.

The computation of the solution can be understood as a typical problem to solve with the Lagrange multipliers method. We want to find the points maximizing the function $\Psi$ under the requirement that $\left(\lambda_{1}, \ldots, \lambda_{n}\right)$ is in the positive part of the unit sphere of $\left(\mathbb{R}^{n}\right)^{+}$, that in this case is given by the restriction

$$
\sum_{i=1}^{n} \lambda_{i}^{2}=1
$$

In order to solve this, just find the solution of the system that appear when we compute the derivatives of the function

$$
\Gamma\left(\lambda_{1}, \ldots, \lambda_{n}\right):=\Psi\left(\lambda_{1}, \ldots, \lambda_{n}\right)-\gamma\left(\lambda_{1}^{2}+\ldots+\lambda_{n}^{2}-1\right),
$$

equal to 0 . That is, for $i=1, \ldots, n$ we have that

$$
\begin{aligned}
\frac{\partial \Gamma}{\partial \lambda_{i}} & =\frac{\partial}{\partial \lambda_{i}}\left[\Psi\left(\lambda_{1}, \ldots, \lambda_{n}\right)-\gamma\left(\lambda_{1}^{2}+\ldots+\lambda_{n}^{2}-1\right)\right] \\
& =\frac{\partial}{\partial \lambda_{i}}\left[\left(I\left(A_{1}\right)+\ldots+I\left(A_{m}\right) \mid\left(\lambda_{1}, \ldots, \lambda_{n}\right)\right)\right]-2 \gamma \lambda_{i}=0 \\
& =\left(I\left(A_{1}\right)+\ldots+I\left(A_{m}\right)\right)_{i}-2 \gamma \lambda_{i}=0
\end{aligned}
$$


and so

$$
\lambda_{i}^{2}=\frac{1}{4 \gamma^{2}}\left(I\left(A_{1}\right)+\ldots+I\left(A_{m}\right)\right)_{i}^{2} .
$$

Then, together with (2) and taking into account that the maximum is attained for positive coordinates $\lambda_{i}$, we get

$$
1=\sum_{i=1}^{n} \lambda_{i}^{2}=\frac{1}{4 \gamma^{2}} \sum_{i=1}^{n}\left(I\left(A_{1}\right)+\ldots+I\left(A_{m}\right)\right)_{i}^{2} .
$$

Thus,

$$
2 \gamma=\sqrt{\sum_{i=1}^{n}\left(I\left(A_{1}\right)+\ldots+I\left(A_{m}\right)\right)_{i}^{2}} .
$$

Therefore, for each $i=1, \ldots, n$ we obtain

$$
\lambda_{i}=\frac{\left(I\left(A_{1}\right)+\ldots+I\left(A_{m}\right)\right)_{i}}{\sqrt{\sum_{i=1}^{n}\left(I\left(A_{1}\right)+\ldots+I\left(A_{m}\right)\right)_{i}^{2}}} .
$$

In other words, the determination of the vector valued impact measure that solves our problem is given by the scalar positive impact measure

$$
I_{0}(A)=\sum_{i=1}^{n} \frac{\left(I\left(A_{1}\right)+\ldots+I\left(A_{m}\right)\right)_{i}}{\sqrt{\sum_{i=1}^{n}\left(I\left(A_{1}\right)+\ldots+I\left(A_{m}\right)\right)_{i}^{2}}} I(A)_{i} .
$$

A relevant fact that must be pointed out here is that the optimization method used above for finding the best determination does not depend on the countable additivity of the scalar impact measures involved. This means that the same method can be used, for example, if the h-index is put in one coordinate of the vector valued impact measure constructed here. Of course, in this case the resulting vector valued function is not a vector measure, and so it does not satisfy the properties of such functions, but the optimal character of the obtained scalar impact measure among the corresponding family of scalar functions still remains.

4.1. Newspapers Impact Factor. Let us finish this section by explaining our procedure by means of a non standard example, in which non atomic measures are needed. The reason is that the index is defined by using the value of the area of pieces of paper, and so Lebesgue measure in $\mathbb{R}^{2}$ is needed. We will give a specific vector valued impact factor for measuring the impact of news in newspapers, with a determination for the case of news about sports.

Let us give a simple example of how our technique can be used. Suppose that we want to measure the social impact that two football players $p_{1}$ and $p_{2}$ have in a given country in a given year by means of the news appearing in three newspapers $N_{1}, N_{2}$ and $N_{3}$ of similar size that are published in the year. We propose to measure the impact of news or sets of news as the physical area that it occupies in the newspapers in all the year. Therefore, we define $\Omega$ to be the total area of published pages in the newspapers, and the associated measure space $(\Omega, \Sigma, \mu)$ to be Lebesgue measure space in $\Omega \subset \mathbb{R}^{2}$. Write $\Omega_{1}, \Omega_{2}$ and $\Omega_{3}$ to be the subsets of $\Omega$ that represent the total published (physical) areas in each of the three journals. 
The vector valued impact factor $N$ is then defined as

$$
N(A):=\sum_{i=1}^{3} \frac{\mu\left(\Omega_{i} \cap A\right)}{\mu(A)} e_{i} \in \mathbb{R}^{3}, \quad A \in \Sigma .
$$

The journals are supposed to be general, that is, publishing general news and not only news about sports. However, the scopes are different, so the area devoted to reports on sports is different in each of them. Since we are talking about football players, it seems natural to give a bigger weight in the final computation to the journals that devote more space to sports. That is, the determination must be given by the vector

$$
v_{S}:=\frac{1}{\mu(D)}\left(\mu\left(\Omega_{1} \cap D\right), \mu\left(\Omega_{2} \cap D\right), \mu\left(\Omega_{3} \cap D\right)\right),
$$

where $D$ is the set of $\Sigma$ given by all the areas of the reports on sports in the three journals.

Thus, the final (scalar) impact factor obtained in this way is

$$
N_{0}(A)=\sum_{i=1}^{3} \frac{\mu\left(\Omega_{i} \cap D\right)}{\sqrt{\sum_{i=1}^{3} \mu\left(\Omega_{i} \cap D\right)^{2}}} \frac{\mu\left(\Omega_{i} \cap A\right)}{\mu(A)} .
$$

Therefore, if $A_{i}$ is given by the (areas occupied by the) news devoted to $p_{i}, i=1,2$, then $N_{0}\left(A_{1}\right)>N_{0}\left(A_{2}\right)$ means that $p_{1}$ has more social impact than $p_{2}$.

This example shows also that proper (non atomic) measures - and not only the counting measure - may be taken into account in the performance of a general information impact theory. Lebesgue measure is necessary in this case, since it provides the best measure of the printed areas. More examples can be found in Ferrer et al. (2015).

\section{How to PERFORM A quANTItative SELECTION TOOL FOR A RESEARCH INSTITUTE}

The next procedure is performed for giving a solution to one of the topics of interest in research assessment: how to create a numerical suitable impact factor for a concrete research institute for evaluating the curricula of several candidates for a research position.

We want to define an index for evaluating the best candidate for a research contract for a certain research institute. In order to do that, we want to use the values of the impact factor $\mathrm{IF}_{2}$ of the journals appearing in the corresponding list in all the period of years from 1997 to 2013. Each of these indexes can be used for giving a weight to the citations of the papers published by each candidate in the last 5 years, that is the period of time that has been considered as convenient by the institute.

The adequate mathematical structure can be defined as follows. Let $\Omega$ be the set of all the papers published by all the candidates during the last 5 years. $\Sigma$ is the class of the all the subsets of $\Omega$. Note that maybe there are more than one candidate for the same paper, since it can happen that some of them are scientific collaborators. We consider $\mu$ to be the counting measure on the subsets of $\Omega$, that is defining by associating to each subset its cardinal. A candidate $p$ has a set of papers $A(p) \in \Sigma$, the ones in which he appears as an author. As we said, it may happen that $A\left(p_{1}\right) \cap A\left(p_{2}\right) \neq \emptyset$ for $p_{1} \neq p_{2}$. 
The weighted vector valued impact measure is given by some weights that must be defined by means of an impact factor type measure. To be coherent, the weights must be related to the order that a given journal has in the list and not to the numerical value of the 2-year impact factor in a year. Also, all the weights must be normalized to 1 in order to let each year to have the same weight in the final sum. Therefore, we will use the order ranking $\mathrm{RN}_{2}$ of the journal in the JCR list in a given year normalized to the interval $[0,1]$, instead of the value of $\mathrm{IF}_{2}$ of the journal. This index was defined in Pudovkin and Garfield (2004) with the name Rank-normalized impact factor.

Therefore, the vector valued impact measure must be defined as

$$
R(A):=\frac{1}{\mu(A)} \int_{A}\left(\sum_{i=1}^{17} \mathrm{RN}_{2}^{i}(a) e_{i}\right) d \mu(a),
$$

that is; for each $i=1, \ldots, 17$, the scalar weights $\mathrm{RN}_{2}^{i}(a)$ given to each paper $a$ in each coordinate $i$, is the 2-year rank-normalized impact factor $\mathrm{RN}_{2}$ of the journal where $a$ is published, in each year of the time series $1997-2013$. In the same way, if $r$ is a journal, we write $\mathrm{RN}_{2}^{i}(r)$ for the rank-normalized impact factor of $r$ in the year indexed by $i$.

Using this vector valued impact factor, we will define now a scalar impact factor according to the institute preferences. The scientific policy of the institute wants to benefit the authors publishing papers in a given specific field of mathematics, for example, Banach lattices. However, the ordering given by the corresponding impact lists and the rest of the mathematical subjects must be also taken into account, and publications in good journals must be still providing good evaluations.

The way of performing the weights following these ideas is the following. The institute chooses an adequate set of reference journals that publishes papers on Banach lattices. It seems adequate to give a better weight in the final evaluation of the impact of a given paper to the rank normalized impact factors of the years in which the selected set of Banach lattice journals have bigger values of this index. In a sense, the list of these years are giving more relevance to the topic Banach lattices, and so they must be more important for evaluating the curricula of the candidates.

Thus, choose the journals $r_{1}, \ldots, r_{n}$ that the institute considers relevant for evaluation, and write $A\left(r_{j}\right) \in \Sigma, j=1, \ldots, n$, for the sets of papers in $\Omega$ that have been published in the journal $r_{j}$. This will be the set of subsets that will be used for the optimization process. Clearly, for each $j$ we have that

$$
R\left(A\left(r_{j}\right)\right)=\frac{1}{\mu\left(A\left(r_{j}\right)\right)}\left(\sum_{i=1}^{17} \operatorname{RN}_{2}^{i}\left(r_{j}\right) e_{i}\right) \mu\left(A\left(r_{j}\right)\right)=\sum_{i=1}^{17} \operatorname{RN}_{2}^{i}\left(r_{j}\right) e_{i} .
$$

Thus, we get the weighted scalar index RW, that is given by the formula

$$
\begin{aligned}
\operatorname{RW}(A) & =\frac{1}{\mu(A)} \sum_{a \in A} \sum_{i=1}^{17} \mathrm{RN}_{2}^{i}(a) \frac{\left(R\left(A\left(r_{1}\right)\right)+\ldots+R\left(A\left(r_{n}\right)\right)\right)_{i}}{\sqrt{\sum_{i=1}^{17}\left(R\left(A\left(r_{1}\right)\right)+\ldots+R\left(A\left(r_{n}\right)\right)_{i}^{2}\right.}} \\
& =\frac{1}{\mu(A)} \sum_{a \in A} \sum_{i=1}^{17} \operatorname{RN}_{2}^{i}(a) \frac{\mathrm{RN}_{2}^{i}\left(r_{1}\right)+\ldots+\mathrm{RN}_{2}^{i}\left(r_{n}\right)}{\sqrt{\sum_{i=1}^{17}\left(\mathrm{RN}_{2}^{i}\left(r_{1}\right)+\ldots+\mathrm{RN}_{2}^{i}\left(r_{n}\right)\right)^{2}}},
\end{aligned}
$$


for each $A \subseteq \Omega$. If the set $A$ contains a unique element $a$, we write $\operatorname{RW}(a)$ instead of $\operatorname{RW}(A)$. Thus, for computing the mark $M(p)$ that the method assigns to a candidate $p$, is enough to consider the contributions of all journals in $A(p)$, that is

$$
M(p)=\sum_{a \in A(p)} \operatorname{RW}(a)
$$

Let us give a numerical example consisting of two parts. The difference among them is the set of Banach lattice journal that is considered.

\section{A set of Banach lattice journals defined by four elements}

Assume first, just for the example, that the values of the rank normalized impact factors from 1997 to 2013 of the journals that the institute considers to be the best journals publishing on the subject Banach lattices are:

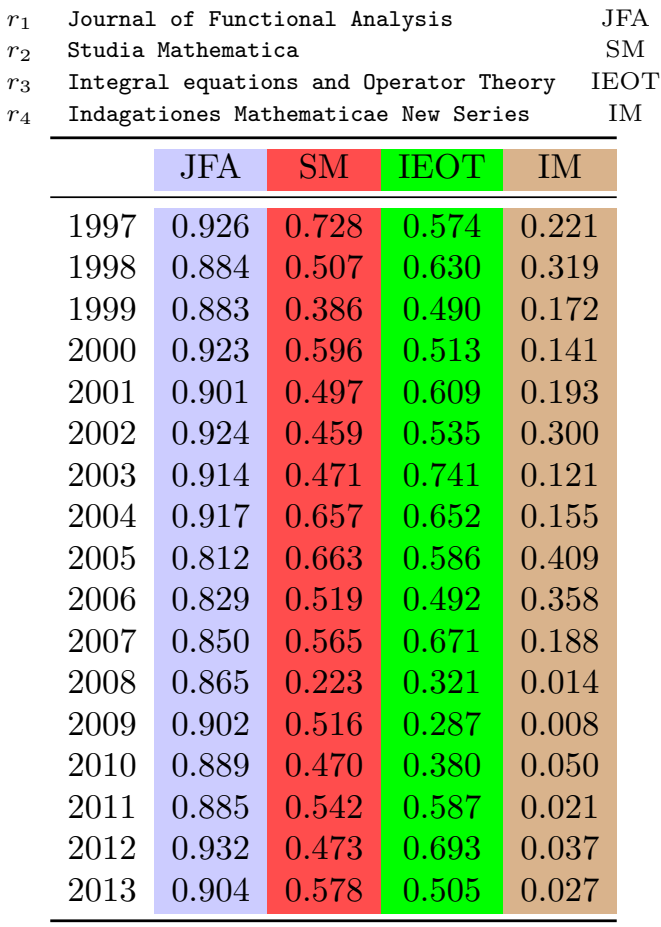

The following graphic shows the evolution of the values of the rank normalized impact factors of the journals in the time period considered. 


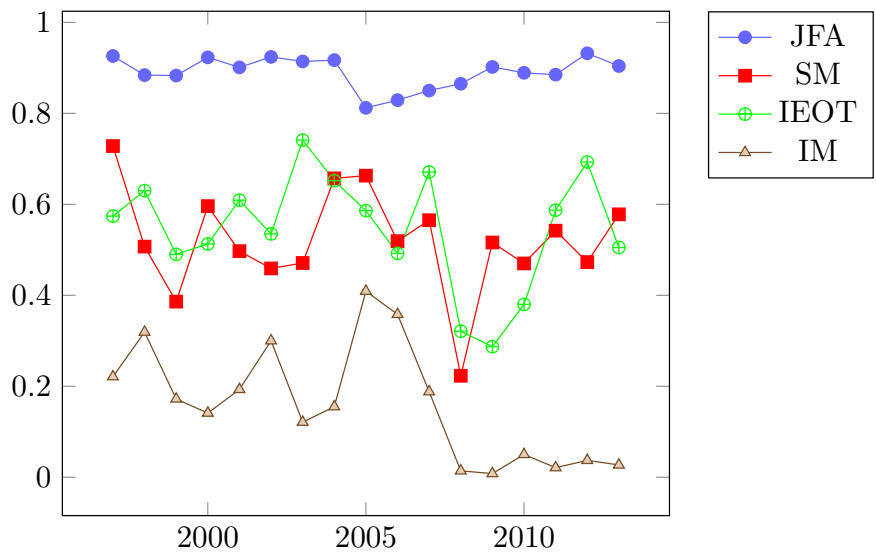

$\mathrm{RN}_{2}$ of the journals considered for the definition of the weights.

After making the adequate computations, the final weights that we obtain when these journals are considered, that are given by the formula

$$
\lambda_{i}=\frac{\mathrm{RN}_{2}^{i}\left(r_{1}\right)+\ldots+\mathrm{RN}_{2}^{i}\left(r_{4}\right)}{\sqrt{\sum_{i=1}^{17}\left(\mathrm{RN}_{2}^{i}\left(r_{1}\right)+\ldots+\mathrm{RN}_{2}^{i}\left(r_{4}\right)\right)^{2}}}
$$

for $i=1, \ldots, 17$, are presented in the following figure:

\begin{tabular}{ll}
\hline 1997 & 0.278 \\
1998 & 0.266 \\
1999 & 0.219 \\
2000 & 0.246 \\
2001 & 0.249 \\
2002 & 0.252 \\
2003 & 0.255 \\
2004 & 0.270 \\
2005 & 0.280 \\
2006 & 0.249 \\
2007 & 0.258 \\
2008 & 0.161 \\
2009 & 0.194 \\
2010 & 0.203 \\
2011 & 0.231 \\
2012 & 0.242 \\
2013 & 0.228 \\
\hline
\end{tabular}

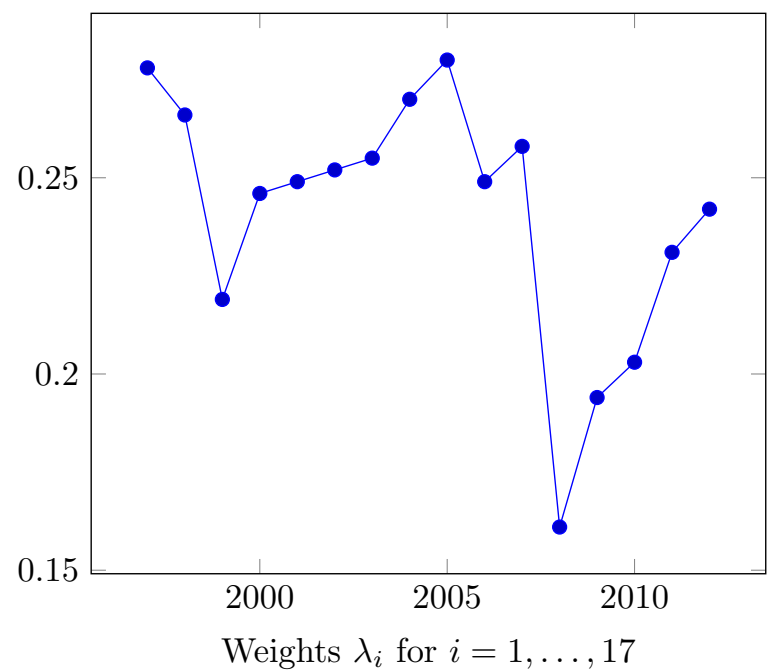

Suppose that we have three candidates presenting their curricula to the research institute, and that they have the following publications in the last 5 years: 


\begin{tabular}{lll}
\hline Candidate 1 & 1 paper in & Inventiones Mathematicae \\
& 1 paper in & Constructive Approximation \\
& 1 paper in & Studia Mathematica \\
& 1 paper in & Journal of Algebra \\
\hline Candidate 2 & 1 paper in & Annals of Mathematics \\
& 2 papers in & Acta Arithmetica \\
& 1 paper in & Integral Equations and Operator Theory \\
\hline Candidate 3 & 11 papers in & Indagationes Mathematicae \\
\hline
\end{tabular}

The corresponding values of the rank normalized impact factors of these journals are presented of each candidate are presented in the next tables and figures.

\begin{tabular}{lllll}
\hline 1997 & 0.985 & 0.809 & 0.728 & 0.581 \\
1998 & 0.949 & 0.928 & 0.507 & 0.696 \\
1999 & 0.959 & 0.952 & 0.386 & 0.669 \\
2000 & 0.974 & 0.904 & 0.596 & 0.660 \\
2001 & 0.963 & 0.950 & 0.497 & 0.671 \\
2002 & 0.965 & 0.888 & 0.459 & 0.641 \\
2003 & 0.960 & 0.730 & 0.471 & 0.603 \\
2004 & 0.983 & 0.729 & 0.657 & 0.691 \\
2005 & 0.967 & 0.878 & 0.663 & 0.497 \\
2006 & 0.979 & 0.898 & 0.519 & 0.588 \\
2007 & 0.961 & 0.966 & 0.565 & 0.657 \\
2008 & 0.977 & 0.940 & 0.223 & 0.586 \\
2009 & 0.988 & 0.949 & 0.516 & 0.492 \\
2010 & 0.978 & 0.968 & 0.470 & 0.548 \\
2011 & 0.983 & 0.892 & 0.542 & 0.563 \\
2012 & 0.983 & 0.892 & 0.473 & 0.520 \\
2013 & 0.983 & 0.907 & 0.578 & 0.532 \\
\hline
\end{tabular}

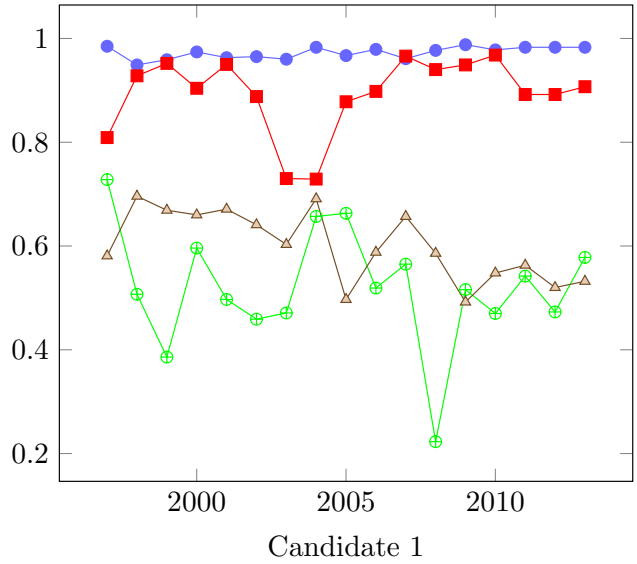

\begin{tabular}{|c|c|c|c|}
\hline 1997 & 1.000 & 0.757 & 0.574 \\
\hline 1998 & 0.986 & 0.754 & 0.630 \\
\hline 1999 & 1.000 & 0.703 & 0.490 \\
\hline 2000 & 0.968 & 0.500 & 0.513 \\
\hline 2001 & 0.988 & 0.627 & 0.609 \\
\hline 2002 & 0.988 & 0.606 & 0.535 \\
\hline 2003 & 0.971 & 0.586 & 0.741 \\
\hline 2004 & 0.978 & 0.470 & 0.652 \\
\hline 2005 & 0.994 & 0.315 & 0.586 \\
\hline 2006 & 0.995 & 0.267 & 0.492 \\
\hline 2007 & 1.000 & 0.319 & 0.671 \\
\hline 2008 & 0.991 & 0.340 & 0.321 \\
\hline 2009 & 1.000 & 0.331 & 0.287 \\
\hline 2010 & 0.993 & 0.362 & 0.380 \\
\hline 2011 & 0.990 & 0.316 & 0.587 \\
\hline 2012 & 0.993 & 0.351 & 0.693 \\
\hline 2013 & 0.993 & 0.266 & 0.505 \\
\hline
\end{tabular}

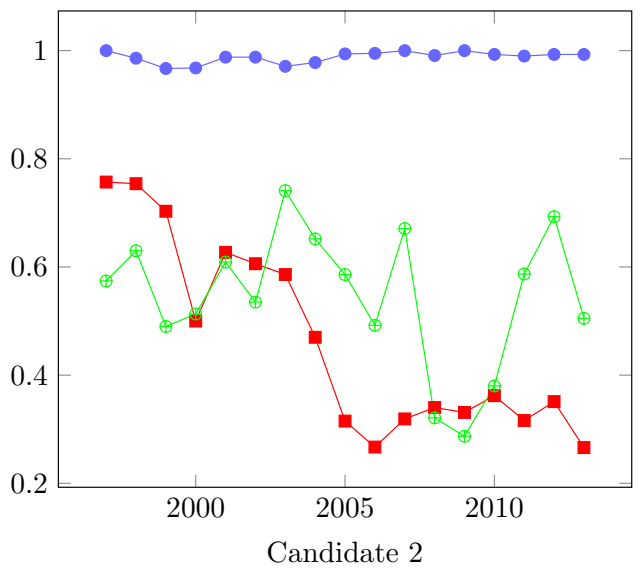



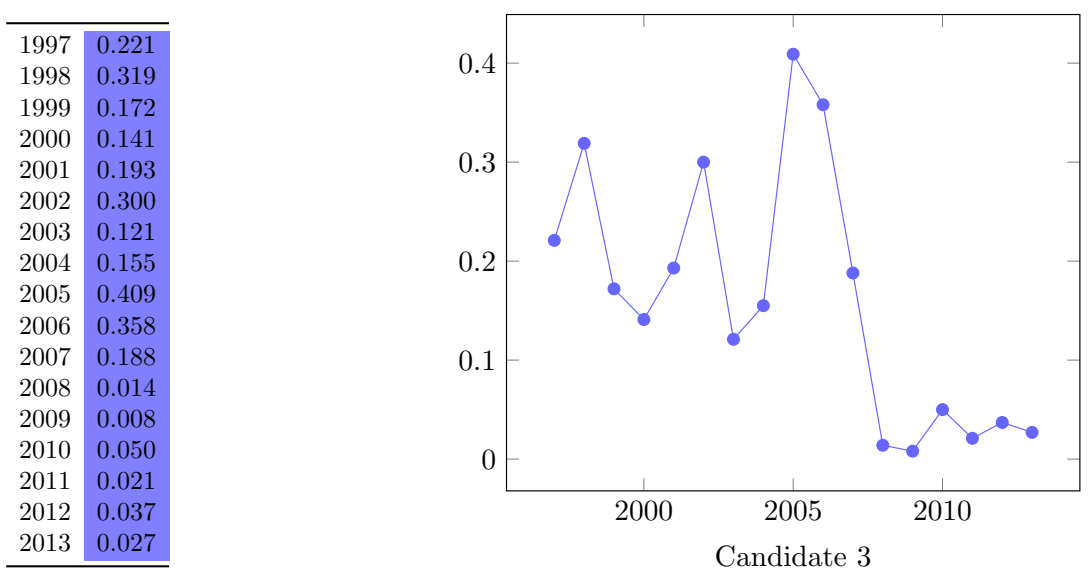

The marks obtained for each candidate using the formulas explained above are:

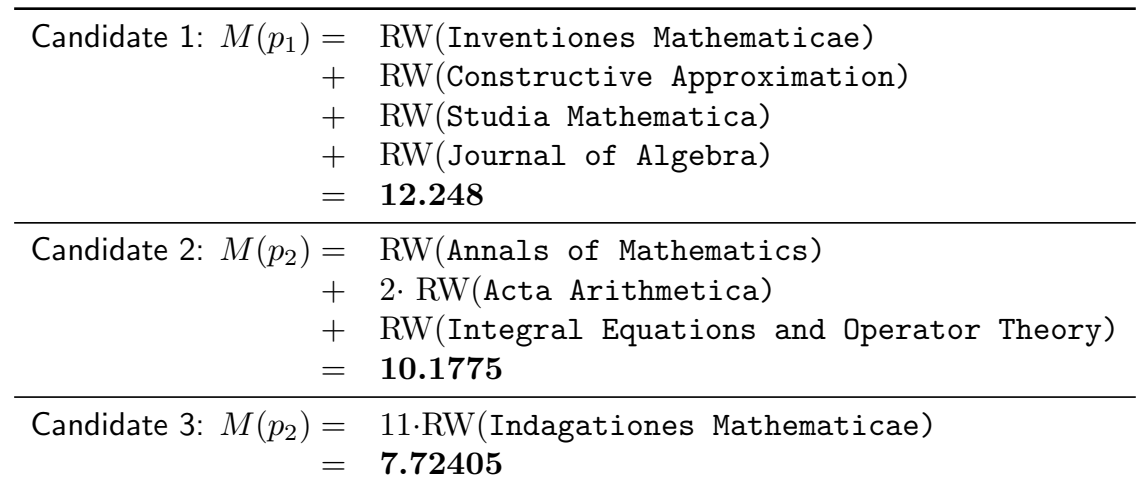

The journal Indagationes Mathematicae is the one with lowest values among all the four journals considered for defining the set of Banach lattice journals. In what follows we compare the relative weight that our model gives to the journals appearing in the curricula of the candidates when compared to the one of Indagationes Mathematicae.

The ratio among the media $\overline{\mathrm{RN}_{2}}$ of the values of the $\mathrm{RN}_{2}$ of some journals in the 17 years time series with respect to the media of Indagationes Mathematicae is given in the first column of the next table. The second one presents the values of the ratio among the values of RW of each journal and the one of Indagationes Mathematicae.

\begin{tabular}{ccc}
\hline & Ratio $\overline{\mathrm{RN}_{2}}$ & Ratio RW \\
\hline Inventiones Mathematicae & 6.05 & 5.66 \\
Constructive Approximation & 5.55 & 5.17 \\
Studia Mathematica & 3.24 & 3.09 \\
Acta Arithmetica & 2.88 & 2.74 \\
\hline
\end{tabular}

This ratio measures the relevance of each journal with respect to the one that is token as reference, Indagationes Mathematicae. The reader can notice that the ratio is smaller when the new weights are considered. That is, Indagationes Mathematicae, that is a journal in the selected list of journals of Banach lattices, is more important when the new weight is considered. This behavior will be much 
more relevant in the next example, in which the set of Banach lattice journals is defined just by Indagationes Mathematicae as the only element.

\section{A set of Banach lattice journals defined by a unique journal}

In this section we repeat the computation of the marks for the same candidates of the previous section in the case that the weights for defining RW are the $\mathrm{RN}_{2}$ values of Indagationes Mathematicae in all the years. The procedure of computation is the same, by the final result are clearly different.

In this case, we obtain the following marks.

\begin{tabular}{|c|c|}
\hline Candidate $1: M\left(p_{1}\right)=$ & $\begin{array}{l}=\text { RW(Inventiones Mathematicae) } \\
+ \text { RW(Constructive Approximation) } \\
+ \text { RW(Studia Mathematica) } \\
+ \text { RW(Journal of Algebra) } \\
=\mathbf{9 . 8 5 4}\end{array}$ \\
\hline Candidate 2: $M\left(p_{2}\right)=$ & $\begin{array}{l}=\text { RW(Annals of Mathematics) } \\
+\quad 2 \cdot \text { RW(Acta Arithmetica) } \\
+ \text { RW(Integral Equations and Operator Theory) } \\
=\mathbf{8 . 4 0 5}\end{array}$ \\
\hline Candidate $3: \begin{aligned} M\left(p_{2}\right) & = \\
& =\end{aligned}$ & $\begin{array}{l}=11 \cdot \mathrm{RW}(\text { Indagationes Mathematicae }) \\
=\mathbf{9 . 2 1 5}\end{array}$ \\
\hline
\end{tabular}

The reader can notice that the order provided by this evluation is different than the one obtained in the previous section. In this case, Candidate 3 - the one that has more papers in a journal with a relevant amount of publications in Banach lattices, but with small impact- gets better marks than Candidate 2. However, Candidate 1 still wins. The relative weight of Indagationes Mathematicae can be seen in the following table.

\begin{tabular}{ccc}
\hline & Ratio $\overline{\mathrm{RN}_{2}}$ & Ratio RW \\
\hline Inventiones Mathematicae & 6.05 & 3.77 \\
Constructive Approximation & 5.55 & 3.45 \\
Studia Mathematica & 3.24 & 2.14 \\
Acta Arithmetica & 2.88 & 1.97 \\
\hline
\end{tabular}

Clearly, Indagationes Mathematicae has much better marks in comparison to the other journals.

\section{Conclusions}

In order to provide a response to the increasing interest in using several impact indexes (altmetrics) together for research assessment, we show a suitable mathematical structure based on finite dimensional (vector) valued information measures or generalized impact factors. We have introduced a mathematical representation of such an instrument, describing the way a concrete determination of the vector valued measure can be obtained in order to provide an adequate (scalar valued) impact factor. This new index is defined to be the best for representing a previously fixed evaluation criterium, and it is obtained as a result of an optimization process. In other words, we show that, when we fix a finite set of impact measures, a previously established criterium about which are the more relevant elements in the evaluation process provides a unique new scalar index that is exactly the most 
adequate for our aim. For example, we show that this allows to perform a specific suitability index for individual scientific curricula in order to find the most adequate for a research position in a scientific institute or a company.

The main restriction of the model is that all the scalar measures involved in the definition of the vector valued impact measure must be themselves countably additive. Then, for example in the actual model the Hirsch index cannot be used, since it is not additive. However, some modifications using non additive measure theory (integration with respect to capacities or fuzzy measures) should solve the problem for providing a more general framework.

\section{REFERENCES}

[1] Aleixandre Benavent, R., Valderrama Zurián, J.C., González Alcaide, G. (2007). Scientific journals impact factor: limitations and alternative indicators. El Profesional de la Información, 16(1), 4-11.

[2] Buela-Casal, G. (2003). Evaluating quality of articles and scientific journals. Proposal of weighted impact factor and a quality index. Psicothema, 15(1), 23-25.

[3] Ferrer-Sapena, A., Sánchez-Pérez, E. A., González, L. M., Peset, F. and Aleixandre-Benavent, R. (2015). The impact factor as a measuring tool of the prestige of the journals in research assessment in mathematics. Accepted for publication in Research Evaluation.

[4] Egghe, L., Rousseau, R. (2002). A general frame-work for relative impact indicators. Canadian Journal of Information and Library Science, 27(1), 29-48.

[5] Leydesdorff, L., Opthof, T. (2010). Scopus's Source Normalized Impact per Paper (SNIP) Versus a Journal Impact Factor Based on Fractional Counting of Citations. Journal of the American Society for Information Science and Technology, 61, 2365-2369.

[6] Piwowar, H. (2013). Altmetrics: Value all research products. Nature, 493(7431), 159-159.

[7] Zahedi, Z., Costas, R., Wouters, P. (2014). How well developed are altmetrics? A crossdisciplinary analysis of the presence of 'alternative metrics' in scientific publications. Scientometrics, 101(2), 1491-1513.

[8] Gagolewski, M., Mesiar, R., (2014). Monotone measures and universal integrals in a uniform framework for the scientific impact assessment problem. Information Sciences, 263, 166-174.

[9] Klement, E., Mesiar, R., Pap, E., (2010). A universal integral as common frame for Choquet and Sugeno integral. IEEE Trans. Fuzzy Syst. 18, 178-187.

[10] Torra, V., Narukawa, Y., (2008). The h-index and the number of citations: two fuzzy integrals. IEEE Trans. Fuzzy Syst. 16, 795-797.

[11] Beliakov, G., James, S. (2011). Citation-based journal ranks: the use of fuzzy measures. Fuzzy Sets and Systems, 167, 101-119.

[12] Ferrer-Sapena, A., Sánchez-Pérez, E. A., González, L. M., Peset, F. and Aleixandre-Benavent, R. (2015). Mathematical properties of weighted impact factors based on measures of prestige of the citing journals. Scientometrics, 105(3), 2089-2108.

[13] Alguliyev, R., Aliguliyev, R. and Ismayilova, N. (2015). Weighted Impact Factor (WIF) for assessing the quality of scientific journals. arXiv:1506.02783.

[14] Habibzadeh, F., Yadollahie, M. (2008). Journal weighted impact factor: A proposal. Journal of Informetrics, 2(2), 164-172.

[15] Waltman, L., van Eck, N.J. (2008). Some comments on the journal weighted impact factor proposed by Habibzadeh and Yadollahie. Journal of Informetrics, 2(4), 369-372.

[16] Zitt, M, Small, H. (2008). Modifying the journal impact factor by fractional citation weighting: The audience factor. Journal of the American Society for Information Science and Technology, 59, 1856-1860.

[17] Zyczkowski, K. (2010). Citation graph, weighted impact factors and performance indices. Scientometrics, 85(1), 301-315.

[18] Pinski, G, Narin, F. (1976). Citation influence for journal aggregates of scientific publications: theory, with application to the literature of physics. Information Processing and Management, 12, 297-312.

[19] Diestel , J., Uhl, J.J. Vector measures, Am. Math. Soc., Providence, 1977.

[20] Moed, H.F. (2010). Measuring contextual citation impact of scientific journals. Journal of Informetrics, 4, 265-277. 
[21] Zitt, M. (2011). Behind citing-side normalization of citations: some properties of the journal impact factor. Scientometrics, 89, 329-344.

[22] Dorta-González, P., Dorta-González, M.I. (2013). Comparing journals from different fields of science and social science through a JCR subject categories normalized impact factor. Scientometrics, 95(2), 645-672.

[23] Dorta-González, P., Dorta-González, M.I., Santos-Penate, D.R., Suarez-Vega, R. (2014). Journal topic citation potential and between-field comparisons: The topic normalized impact factor, Journal of Informetrics, 8(2), 406-418.

[24] Li, Y.R., Radicchi, F., Castellano, C., Ruiz-Castillo, J. (2013). Quantitative evaluation of alternative field normalization procedures. Journal of Informetrics, 7(3), 746-755.

[25] Owlia, P., Vasei, M., Goliaei, B., Nassiri, I. (2011). Normalized impact factor (NIF): An adjusted method for calculating the citation rate of biomedical journals. Journal of Biomedical Informatics, 44(2), 216-220.

[26] Ruiz Castillo, J., Waltman, L. (2015). Field-normalized citation impact indicators using algorithmically constructed classification systems of science. Journal of Informetrics, 9, 102117.

[27] Beauzamy, B. Introduction to Banach spaces and their geometry. North-Holland. Amsterdam. 1982.

[28] Pudovkin,A.I., Garfield, E. (2004). Rank-normalized impact factor: A way to compare journal performance across subject categories. Proceedings of the 67th Annual Meeting of the American Society for Information science and technology, 41, 507-515.

J.M. Calabuig. Instituto Universitario de Matemática Pura y Aplicada, UniverSitat Politècnica de València. Camino de Vera S/N, 46022 Valencia, Spain., JMcalABU@MAT.UPV.ES

A. Ferrer-Sapena. Corresponding author. Instituto de Diseño y Fabricación, Universitat Politècnica de València. Camino de Vera S/n, 46022 Valencia, Spain. Tel.: +34963879392, FAX: +34-963879390., ANFERSA@UPV.ES,

E.A. Sánchez-Pérez. Instituto Universitario de Matemática Pura y Aplicada, Universitat Politècnica de València. Camino de Vera s/n, 46022 Valencia, Spain., eaSANCPE@MAT.UPV.ES 